\title{
EFFICIENT SYNTHESIS OF BENZOTHIAZINE AND ACRYLAMIDE COMPOUNDS
}

\author{
Ana Maria Alves Souza, Simone Torres Pádua Walfrido, Lúcia Fernanda Costa Leite, Maria Carmo Alves Lima, \\ Suely Lins Galdino e Ivan Rocha Pitta* \\ Departamento de Antibióticos, Universidade Federal de Pernambuco, 50670-901 Recife - PE, Brasil \\ José Maria Barbosa Filho
}

Universidade Federal da Paraíba, 58051-900 João Pessoa - PB, Brasil

Carlos Alberto De Simone e Javier Alcides Ellena

Instituto de Física, Universidade de São Paulo, 13566-590 São Carlos - SP, Brasil

Recebido em 4/3/09; aceito em 1/9/09; publicado na web em 28/1/10

\begin{abstract}
This article describes the synthesis of the new (2Z)-2-(4-methoxybenzylidene)-6-nitro-4H-benzo[1,4]thiazin-3-one, (2Z)-2-(4methoxybenzylidene)-4-methyl-6-nitro-4H-benzo[1,4]thiazin-3-one, (2Z)-6-amino-2-(4-methoxybenzylidene)- $4 H$-benzo[1,4] thiazin-3-one, (2Z)-6-butylamino-2-(4-methoxybenzylidene)-4-methyl-4H-benzo[1,4]-thiazin-3-one and (2E)- $N$-alkyl- $N$-(2hydroxy-5-nitrophenyl)-3-phenylacrylamides and the spectroscopic data. The arylidenebenzothiazine compounds were prepared using the Knoevenagel condensation with substituted benzaldehydes in the presence of sodium methoxide in DMF. The presence of a nitro substituent in the 4-position, water and a slightly acid reaction medium in this condensation caused the rupture of the benzothiazine ring and subsequent formation of the phenylacrylamide compounds. A crystallographic data was presented for $(2 E)$ 3-(4-bromophenyl)- $N$-dodecyl- $N$-(2-hydroxy-5-nitrophenyl) acrylamide.
\end{abstract}

Keywords: benzothiazines; acrylamides; X-ray crystallography.

\section{INTRODUCTION}

1,4-Benzothiazine derivatives exhibit a wide range of pharmacological properties, including antifungal, immunostimulating, anti-aldoso-reductase, anti-rheumatic, anti-allergic, vasorelaxant, anti-arrhythmic, anti-hypertensive, neuroprotective and cytotoxic activities. These properties indicate that 1,4-benzothiazine is a template that may be potentially useful in medicinal chemistry research and therapeutic applications. ${ }^{1}$

Gupta and $\mathrm{Ojha}^{2}$ reviewed the chemical and pharmacological properties of phenothiazines and 1,4-benzothiazines. Some of the latter showed microbiological and antitumor activities. ${ }^{3,4}$ The synthesis, spectroscopic properties and microbiological activities of several 6-alkylacylamino-4-methyl-4H-benzo[1,4]thiazin-3-ones have also been reported..$^{5}$ Moreover, the reactivity of a methylene group activated by a carbonyl substituent or a thioxo has been studied by Guarda and Silva. ${ }^{6,7}$ Condensation of 1,4-benzothiazin-3-ones with arylaldehydes or Michael addition on cyanoacrylates leads to the formation of 2-arylidene- $4 H$-benzo[1,4]thiazin-3-one compounds. ${ }^{8}$

\section{RESULTS AND DISCUSSION}

The present study describes the synthesis of substituted (2E)- $N$ alkyl- $N$-(2-hydroxy-5-nitrophenyl)-3-phenylacrylamides, (4-6), (2Z)-2(4-methoxybenzylidene)-6-nitro-4H-benzo[1,4]thiazin-3-one, (7), (2Z)2-(4-methoxybenzylidene)-4-methyl-6-nitro-4H-benzo[1,4]thiazin-3one (8) and (2Z)-6-amino-2-(4-methoxybenzylidene)-4H-benzo[1,4] thiazin-3-one (9) or (2Z)-6-butylamino-2-(4-methoxybenzylidene)-4methyl-4H-benzo[1,4]thiazin-3-one (12), Figure 1 . The intermediary 6-nitro-4H-benzo[1,4]-oxazin-3-one, which were not isolated, were obtained as a result of the Knoevenagel condensation of activated 4-alkyl-6-nitro-4H-benzo[1,4]thiazin-3-one 2-3 with 4-methoxy or 4-bromobenzaldehydes in the presence of sodium methoxide in DMF. ${ }^{9}$

*e-mail: irpitta@gmail.com
The (2Z)-2-(4-methoxybenzylidene)-4-methyl-6-nitro-4H-benzo[1,4] thiazin-3-one 8 was first condensed in position 2; subsequently, alkylation was carried out at position $4 .{ }^{10}$ Methylation followed by condensation did not yield good results. The reduction of the nitro group to a primary amine with tin chloride in concentrated hydrochloric acid produced (2Z)-6-amino-2-(4-methoxybenzylidene)-4H-benzo[1,4] thiazin-3-one 9 or 6-amino-4-methyl-4H-benzo[1,4]thiazin-3-one $\mathbf{1 0} .^{11}$

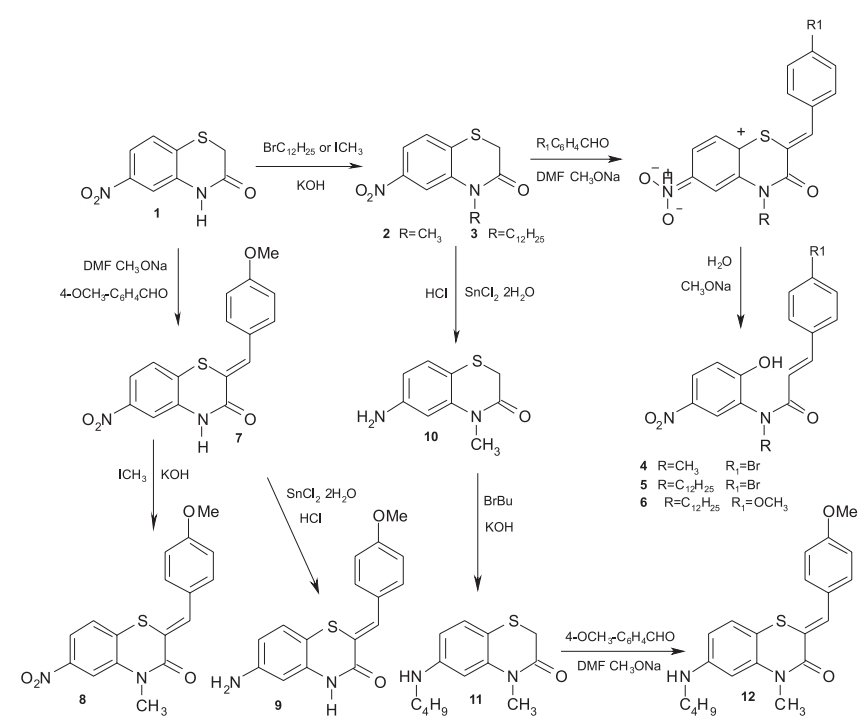

Figure 1. Benzothiazine and acrylamide derivatives: synthetic pathways

The arylidenebenzothiazines $(\mathbf{7 , 1 2})$ described in this work were isolated in a single isomeric form. It has been previously demonstrated ${ }^{12}$ that the $Z$ isomer is the main derivative that is obtained from the standard Knoevenagel condensation of benzaldehydes with benzylidene- $4 \mathrm{H}$ benzo[1,4]thiazin-3-ones in an alkaline medium. The study was carried out using coupled NMR ${ }^{13} \mathrm{C}-\mathrm{H}$ spectrometry. The value of the coupling 
constant between the ethylene proton and the carbon of the carbonyl group in position 3 verifies the existence of the $Z$ isomer.

The (2E)- $N$-alkyl- $N$-(2-hydroxy-5-nitrophenyl)-3-phenylacrylamides 4-6 were formed due to the presence of the nitro group in the para-position, water and the excess of heat lead in a slightly acid reaction medium to the formation of the intermediary 6-nitro- $4 \mathrm{H}$ benzo[1,4]-oxazin-3-one and posterior rupture, figure 2 . The chemical shifts and coupling constants in the ${ }^{1} \mathrm{H}$ NMR of ethylenic protons and the crystallographic data show that the $N$-alkyl- $N$-(2-hydroxy5-nitro-phenyl)-3-phenylacrylamides (4-6) that formed have the $E$ configuration.

The spectroscopic characteristics and IR, ${ }^{1} \mathrm{H}$ NMR and MS spectroscopic data of the arylidenebenzothiazines and acrylamides that were prepared are given in the experimental section.

\section{Crystallographic data}

The crystal data, collection procedures, structure determination methods and refinement results for the (2E)-3-(4-bromo-phenyl)- $N$ dodecyl- $N$-(2-hydroxy-5-nitro-phenyl)acrylamide (5) are summarized in Table 1. We used the following programs: for the cell determination and data collection, ${ }^{13}$ data reduction, ${ }^{14}$ data collection, ${ }^{15}$ structure resolution, ${ }^{16}$ refinement, ${ }^{17}$ graphic presentation ${ }^{18}$ and material to publication. ${ }^{19}$

The structure was solved by direct methods and refined by a fullmatrix least squares calculations. The refinement was conducted until all atomic parameter shifts were smaller than their standard deviations. All $\mathrm{H}$ atoms were located by geometrical considerations. In the final difference Fourier map there are no peaks greater than $0.34 \AA^{-3}$. The asymmetrical unit consists of two independent molecules, which are not related one to the other by symmetry.

Table 1. Crystal data and structure refinement

\begin{tabular}{|c|c|}
\hline Empirical formula & $\mathrm{C}_{27} \mathrm{H}_{35} \mathrm{O}_{4} \mathrm{~N}_{2} \mathrm{Br}$ \\
\hline Formula weight & 531.48 \\
\hline Temperature & $120(2) \mathrm{K}$ \\
\hline Wavelength & $0.71070 \AA$ \\
\hline Crystal system & Monoclinic \\
\hline Space group & $\mathrm{P} 2_{1}$ \\
\hline Unit cell dimensions & $a=11.0421(5) \AA$ \\
\hline$b=7.3059(3) \AA$ & $\beta=91.51(2)^{\circ}$ \\
\hline \multicolumn{2}{|l|}{$c=32.3832(16) \AA$} \\
\hline Volume & $2611.5(2) \AA^{3}$ \\
\hline $\mathrm{Z}$ & 4 \\
\hline Density (calculated) & $1.352 \mathrm{Mg} / \mathrm{m}^{3}$ \\
\hline Absorption coefficient & $1.608 \mathrm{~mm}^{-1}$ \\
\hline $\mathrm{F}(000)$ & 1112 \\
\hline Crystal size & $0.12 \times 0.10 \times 0.10 \mathrm{~mm}$ \\
\hline Theta range for data collection & 1.26 to $25.0^{\circ}$ \\
\hline Index ranges & $-12 \leq h \leq 13,-8 \leq k \leq 8,-38 \leq l \leq 38$ \\
\hline Reflections collected & 15287 \\
\hline Independent reflections & $8677[\mathrm{R}($ int $)=0.0163]$ \\
\hline Absorption correction & Multiscan \\
\hline Refinement method & Full-matrix least-squares on $\mathrm{F}^{2}$ \\
\hline Data / parameters & $5425 / 615$ \\
\hline Goodness-of-fit on $\mathrm{F}^{2}$ & 1.046 \\
\hline Final $R$ indices $[\mathrm{I}>2 \sigma(\mathrm{I})]$ & $\mathrm{R} 1=0.061, \mathrm{wR} 2=0.1191$ \\
\hline
\end{tabular}

In both independent molecules, no conformational differences are observed. Bond lengths and angles agree well, within the experimental accuracy, with values found in literature, Figure 2.

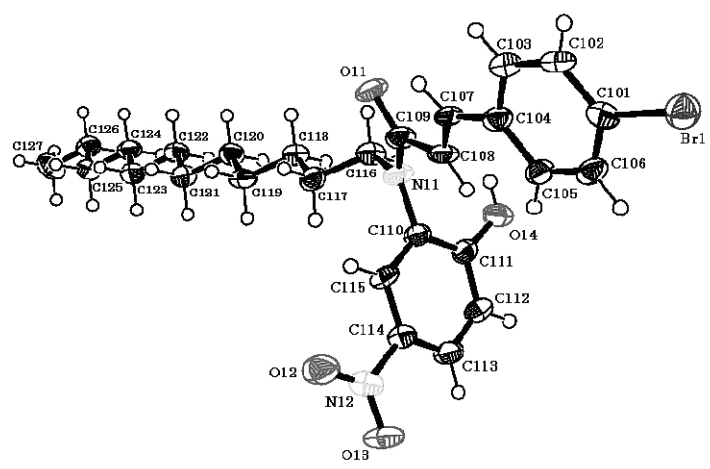

Figure 2. Perspective view of one molecule from the asymmetric unit of the acrylamide $\mathbf{5}$ with atom-labeling. Displacement of ellipsoids plotted at the $30 \%$ probability level are shown for the non- $H$ atoms

\section{CONCLUSION}

The study of the reactivity of 1,4-benzothiazine heterocyclic ring activated in position 3 by carbonyl substituent leads by Knoevenagel condensation with arylaldehydes to the formation of arylidenebenzothiazines compounds in the form of a single isomer in good yields. Phenylacrylamide derivatives were also obtained by rupture of the benzothiazine ring.

\section{EXPERIMENTAL}

Melting points were measured on a Quimis Q340D apparatus with an oil bath in capillary tubes. Thin layer chromatography was performed on Merck $60 \mathrm{~F}_{254}$ silica gel plates with a $0.2 \mathrm{~mm}$ thickness. Compounds were powdered, mixed with $\mathrm{KBr}$ at $1 \%$ concentration and pressed into pellets before infrared spectra were recorded on a FT-IFS 66 Bruker spectrometer; the wavelengths are expressed in $\mathrm{cm}^{-1}$. Nuclear magnetic resonance spectra were recorded on a Varian Unity Plus spectrometer ${ }^{1} \mathrm{H}$ NMR at $300 \mathrm{MHz}$; the chemical shifts $(\delta)$ are expressed in parts per million (ppm) and the coupling constants $(J)$ in Hertz (Hz). DMSO-d was used as the solvent and $\mathrm{SiMe}_{4}$ as the reference. Electronic impact $(70 \mathrm{eV})$ mass spectra were recorded on an HP G1019A, coupled to a CPG HP 5890 spectrometer; for compounds (5) and (6) chemistry ionization was performed in ammonia and isobutane and recorded on a Waters ZQ apparatus. The intensities of the molecular peaks are presented relative to the most intense peak $\mathrm{M}^{+}(\%)$. The synthesis and the spectroscopic characteristics of 6-nitro-4H-benzo[1,4]thiazin-3-one (1), 4-methyl-6-nitro-4H-benzo[1,4]thiazin-3-one (2), 6-amino-4methyl-4H-benzo[1,4]thiazin-3-one (10) and 6-butylamino-4-methyl$4 H$-benzo[1,4] thiazin-3-one (11) have been already described. ${ }^{5}$

\section{4-Dodecyl-6-nitro-4H-benzo[1,4] thiazin-3-one (3)}

A suspension of 6-nitro-4H-benzo[1,4]thiazin-3-one (1) $0.5 \mathrm{~g}(2.38$ $\mathrm{mmol}$ ) in DMSO/EtOH $50 \%$ was treated with potassium hydroxide 0.27 $\mathrm{g}(4.8 \mathrm{mmol})$. The reaction mixture was maintained under strong agitation for $10 \mathrm{~min}$ at room temperature. Dodecylbromide $(1.18 \mathrm{~mL}, 4.76$ mmol) was added dropwise while stirring. The reaction mixture was then maintained under agitation at $55^{\circ} \mathrm{C}$ for $15 \mathrm{~h}$. The crude product was extracted with ethyl acetate. After solvent evaporation, the compound was purified using flash chromatography on silica 60 (230-400 Mesh) using toluene as the eluent. The product appeared as a viscous oil that crystallized after a few days in a desiccator. $\mathrm{C}_{20} \mathrm{H}_{30} \mathrm{~N}_{2} \mathrm{O}_{3} \mathrm{~S}, 0.57 \mathrm{~g}$ (64\%), mp 37-39 ${ }^{\circ} \mathrm{C}$; TLC silica gel plates type $60 \mathrm{~F}_{254}, \mathrm{R}_{\mathrm{f}}\left(\mathrm{C}_{6} \mathrm{H}_{6}\right) 0.58$; IR (potassium bromide) $v_{\max } \mathrm{cm}^{-1}: 2916,2849,1681,1524,1342 \mathrm{~cm}^{-1}$; ${ }^{1} \mathrm{H}$ NMR $\left(300 \mathrm{MHz}, \mathrm{DMSO}-\mathrm{d}_{6}\right): \delta 0.85\left(\mathrm{t}, 3 \mathrm{H}, \mathrm{CH}_{3}\right) \mathrm{J} 6.9 \mathrm{~Hz}, 1.21(\mathrm{~s}$, $\left.16 \mathrm{H}_{2} \mathrm{CH}_{2}\right), 1.51\left(\mathrm{~m}, 2 \mathrm{H}, \mathrm{CH}_{2} \mathrm{CH}_{3}\right), 3.3\left(\mathrm{~m}, 2 \mathrm{H}, \beta \mathrm{CH}_{2}\right), 3.63(\mathrm{~s}, 2 \mathrm{H}$, $\left.\mathrm{SCH}_{2}\right), 4.06$ (t, $\left.2 \mathrm{H}, \alpha \mathrm{CH}_{2}\right) J 7.3 \mathrm{~Hz}, 7.71$ (d, $1 \mathrm{H}, \mathrm{H} 8$ benzothiazine) 
$J 8.4 \mathrm{~Hz}, 7.89$ (dd, $1 \mathrm{H}, \mathrm{H} 7$ benzothiazine) $J 8.4$ and $2.4 \mathrm{~Hz}, 8.02$ (d, 1 $\mathrm{H}$, H5 benzothiazine) $J 2.4 \mathrm{~Hz}$; $\mathrm{MS}, m / z(\%): 378\left(\mathrm{M}^{+} 7.86\right), 331(100)$, 332(9.7), 329(21.9), 211(12.7), 209(3), 195(17.2), 181(27.4).

General procedure for (2E)-N-alkyl-N-(2-hydroxy-5nitrophenyl)-3-phenylacrylamides compounds (4-6)

A mixture of 6-nitro-4-alkyl-4H-benzo[1,4]thiazin-3-one $(0.26$ $\mathrm{mmol}$ ) and substituted benzaldehyde $(0.26 \mathrm{mmol})$ in $3 \mathrm{~mL}$ of DMF was treated with sodium methoxide in excess. The solution was refluxed at $125^{\circ} \mathrm{C}$ for $6 \mathrm{~h}$. After cooling, the isolated precipitate was washed with water. The compounds were of acceptable purity and were analyzed without further recrystallization.

\section{(2E)-3-(4-Bromophenyl)- $N$-(2-hydroxy-5-nitrophenyl)- $N$ - methylacrylamide (4)}

$\mathrm{C}_{16} \mathrm{H}_{13} \mathrm{BrN}_{2} \mathrm{O}_{4}, 0.042 \mathrm{~g}(43 \%), \mathrm{mp} 150-152{ }^{\circ} \mathrm{C}$; TLC silica gel plates type $60 \mathrm{~F}_{254}, \mathrm{R}_{\mathrm{f}}\left(\mathrm{CHCl}_{3}\right.$ : EtOH 99.5:0.5) 0.48; IR (potassium bromide) $v_{\max } \mathrm{cm}^{-1}: 3429,3079,1656,1584,1511,1340,1320,817$; ${ }^{1} \mathrm{H}$ NMR (300 MHz, DMSO-d $): \delta 3.19\left(\mathrm{~s}, 3 \mathrm{H}, \mathrm{CH}_{3}\right), 6.4(\mathrm{~d}, 1 \mathrm{H}$, H2 ethylene) $J 15 \mathrm{~Hz}, 7.13$ (d, 2 H, H2"6" phenyl) $J 9.6 \mathrm{~Hz}, 7.4$ (d, $1 \mathrm{H}, \mathrm{H} 3$ ' phenyl) J 7.5 Hz, 7.49 (d, $2 \mathrm{H}, \mathrm{H} 3$ " 5" phenyl) J 8.4 Hz, 7.5 (d, $1 \mathrm{H}, \mathrm{H} 3$ ethylene) $J 15.6 \mathrm{~Hz}, 8.17$ (s, $1 \mathrm{H}, \mathrm{H} 6$ ' phenyl), 8.18 (d, $1 \mathrm{H}, \mathrm{H} 4$ ' phenyl) J 7.2 Hz; MS, $m / z(\%)$ : 376(M+2.52), 209(100), 210(15.5), 211(88.1), 181(19.6), 102(79.8).

(2E)-3-(4-bromophenyl)- $N$-dodecyl- $N$-(2-hydroxy-5nitrophenyl)acrylamide (5)

$\mathrm{C}_{27} \mathrm{H}_{35} \mathrm{BrN}_{2} \mathrm{O}_{4}, 0.062 \mathrm{~g}(45 \%), \mathrm{mp} 117^{\circ} \mathrm{C}$; TLC silica gel plates type $60 \mathrm{~F}_{254}, \mathrm{R}_{\mathrm{f}}\left(\mathrm{C}_{6} \mathrm{H}_{6}\right.$ : AcOEt 8:2) 0.62; IR (potassium bromide) $v_{\text {max }}$ $\mathrm{cm}^{-1}: 2924,2853,1642,1599,1530,1341,1305 ;{ }^{1} \mathrm{H} \mathrm{NMR}(300 \mathrm{MHz}$, DMSO-d $): \delta 0.84\left(\mathrm{t}, 3 \mathrm{H}, \mathrm{CH}_{3}\right) J 7.2 \mathrm{~Hz}, 1.21\left(\mathrm{~s}, 16 \mathrm{H}, \mathrm{CH}_{2}\right), 1.44$ $\left(\mathrm{m}, 2 \mathrm{H}, \mathrm{CH}_{2} \mathrm{CH}_{3}\right), 3.51\left(\mathrm{~m}, 2 \mathrm{H}, \mathrm{\beta CH}_{2}\right), 3.76\left(\mathrm{~m}, 2 \mathrm{H}, \alpha \mathrm{CH}_{2}\right), 6.34$ (d, $1 \mathrm{H}, \mathrm{H} 2$ ethylene) $J 15.6 \mathrm{~Hz}, 7.09$ (d, $1 \mathrm{H}, \mathrm{H} 3$ ' phenyl) $J 9.3 \mathrm{~Hz}$, 7.38 (d, 2 H, H2" 6" phenyl) J 8.7 Hz, 7.48 (d, 1 H, H3 ethylene) $J 15$ Hz, 7.51 (d, 2 H, H3" '5" phenyl) J 8.4 Hz, 8.06 (d, 1 H, H6' phenyl) $J$ $3 \mathrm{~Hz}, 8.17$ (dd, $1 \mathrm{H}, \mathrm{H} 4$ ' phenyl) $J 9.3$ and $2.6 \mathrm{~Hz}$; DCI, NH $/$ /isobutane: 531 $\left(\mathrm{MH}^{+} 100\right), 532\left(\mathrm{M}+2\right.$ 39.39), 533( $\mathrm{MH}^{+}+2$ 64.49), 397(3.9), 323(21.4), 209(19), 211(15.3), 167(7.2), 130(5.5).

(2E)- $N$-dodecyl- $N$-(2-hydroxy-5-nitrophenyl)-3-(4methoxyphenyl)acrylamide (6)

$\mathrm{C}_{28} \mathrm{H}_{38} \mathrm{~N}_{2} \mathrm{O}_{5}, 0.055 \mathrm{~g}(44 \%), \mathrm{mp} 130-132{ }^{\circ} \mathrm{C}$; TLC silica gel plates type $60 \mathrm{~F}_{254}, \mathrm{R}_{\mathrm{f}}\left(\mathrm{C}_{6} \mathrm{H}_{6}\right.$ :AcOEt 8:2) 0.67; IR (potassium bromide) $\mathrm{v}_{\max }$ $\mathrm{cm}^{-1}: 2926,2854,1645,1604,1513,1336,1250,828 ;{ }^{1} \mathrm{H}$ NMR $(300$ MHz, DMSO-d $)$ ): $\delta 0.84$ (t, $\left.3 \mathrm{H}, \mathrm{CH}_{3}\right) J 6.6 \mathrm{~Hz}, 1.22\left(\mathrm{~s}, 16 \mathrm{H}, \mathrm{CH}_{2}\right)$, $1.42\left(\mathrm{~m}, 2 \mathrm{H}, \mathrm{CH}_{2} \mathrm{CH}_{3}\right), 3.32\left(\mathrm{~m}, 2 \mathrm{H}, \beta_{2} \mathrm{CH}_{2}\right.$ hidden for the water), $3.74\left(\mathrm{~s}, 3 \mathrm{H}, \mathrm{OCH}_{3}\right), 3.79\left(\mathrm{~m}, 2 \mathrm{H}, \alpha \mathrm{CH}_{2}\right), 6.2$ (d, $1 \mathrm{H}, \mathrm{H} 2$ ethylene) $J 15.6 \mathrm{~Hz}, 6.63$ (d, $1 \mathrm{H}, \mathrm{H} 3$ ' phenyl) J $9.9 \mathrm{~Hz}, 6.88$ (d, $2 \mathrm{H}, \mathrm{H} 3$ " 5" phenyl) $J 9 \mathrm{~Hz}, 7.31$ (d, $2 \mathrm{H}, \mathrm{H} 2$ "6" phenyl) $J 8.7 \mathrm{~Hz}, 7.4$ (d, $1 \mathrm{H}$, H3 ethylene) $J 15.6 \mathrm{~Hz}, 7.86$ (d, 1 H, H6' phenyl) $J 3 \mathrm{~Hz}, 7.96$ (dd, $1 \mathrm{H}, \mathrm{H} 4$ ' phenyl) $J 9.1$ and $2.8 \mathrm{~Hz}$; MS, m/z(\%) DCI, $\mathrm{NH}_{3}$ /isobutane: 483( $\left.\mathrm{MH}^{+} 0.95\right), 409(46.6), 384(22.5), 328(20.5), 295(51.2)$, 273(100), 247(29.4), 191(58), 163(68.3).

\section{(2Z)-2-(4-Methoxybenzylidene)-6-nitro-4H-benzo[1,4]thiazin-} 3-one (7)

An equimolar mixture of 6-nitro-4H-benzo[1,4]thiazin-3-one (1) (250 mg, $1.19 \mathrm{mmol})$ and 4-methoxy-benzaldehyde $(162 \mathrm{mg})$ in 2
$\mathrm{mL}$ of anhydrous DMF was treated with sodium methoxide in excess and refluxed at $125^{\circ} \mathrm{C}$ for $3 \mathrm{~h}$ under nitrogen pressure. After cooling, the obtained precipitate was purified by washing with toluene. $\mathrm{C}_{16} \mathrm{H}_{12} \mathrm{~N}_{2} \mathrm{O}_{4} \mathrm{~S}, 0.269 \mathrm{~g}(69 \%)$, mp 261-263 ${ }^{\circ} \mathrm{C}$; TLC silica gel plates type $60 \mathrm{~F}_{254}, \mathrm{R}_{\mathrm{f}}\left(\mathrm{C}_{6} \mathrm{H}_{6}\right.$ :AcOEt 7:3) 0.72; IR (potassium bromide) $\mathrm{v}_{\max }$ $\mathrm{cm}^{-1}: 2956,1665,1604,1512,1343,1255,737 .{ }^{1} \mathrm{H} \mathrm{NMR}(300 \mathrm{MHz}$, DMSO-d $)$ ): $\delta 3.83$ (s, $3 \mathrm{H}, \mathrm{OCH}_{3}$ ), 7.09 (d, $2 \mathrm{H}, \mathrm{H} 3$ ' 5 ' phenyl) $J 9 \mathrm{~Hz}$, 7.68 (d, 2 H, H2' 6' phenyl) $J 8.7$ Hz, 7.6 (d, 1 H, H8 benzothiazine) $J$ $8.7 \mathrm{~Hz}, 7.81$ (s, $1 \mathrm{H}, \mathrm{CH}$ ethylene), 7.82 (dd, $1 \mathrm{H}, \mathrm{H} 7$ benzothiazine) $J$ 8.7 and $2.4 \mathrm{~Hz}, 7.89$ (d, $1 \mathrm{H}, \mathrm{H} 5$ benzothiazine) $J 2.4 \mathrm{~Hz}, 11.24$ (s, 1 H, NH); MS, $m / z(\%): 328\left(\mathrm{M}^{+} 100\right), 313(3.94), 295(84.9), 296(50.7)$, 281(14.1), 249(20).

\section{(2Z)-2-(4-Methoxybenzylidene)-4-methyl-6-nitro-4H- benzo[1,4]thiazin-3-one $(8)$}

A solution of (2Z)-2-(4-methoxybenzylidene)-6-nitro- $4 \mathrm{H}$ benzo[1,4]thiazin-3-one 7 (400 mg, $1.22 \mathrm{mmol}$ ) in DMSO/MEOH $(5 / 6 \mathrm{~mL})$ and potassium hydroxide $(164 \mathrm{mg}, 2.9 \mathrm{mmol})$ was stirred at room temperature for $10 \mathrm{~min}$. Methyl iodide $(0.18 \mathrm{~mL}, 2.43 \mathrm{mmol})$ was added dropwise under stirring. The mixture was stirred at $55^{\circ} \mathrm{C}$ for $15 \mathrm{~h}$. After cooling, the crude was extracted with ethyl acetate. After the solvent evaporated, the compound was washed with acetone. $\mathrm{C}_{17} \mathrm{H}_{14} \mathrm{~N}_{2} \mathrm{O}_{4} \mathrm{~S}, 0.267 \mathrm{~g}(64 \%), \mathrm{mp} 147-150{ }^{\circ} \mathrm{C}$; TLC silica gel plates type $60 \mathrm{~F}_{254},\left(\mathrm{C}_{6} \mathrm{H}_{6}\right) 0.59$, IR (potassium bromide) $\mathrm{v}_{\max } \mathrm{cm}^{-1}$ : $2925,1649,1601,1511,1342,1254,1021,821 ;{ }^{1} \mathrm{H}$ NMR $(300 \mathrm{MHz}$, DMSO-d $)_{6}$ : $\delta 3.56$ (s, $\left.3 \mathrm{H}, \mathrm{CH}_{3}\right), 3.83\left(\mathrm{~s}, 3 \mathrm{H}, \mathrm{OCH}_{3}\right), 7.09$ (d, $2 \mathrm{H}$, H3'5' phenyl) J 8.7 Hz, 7.68 (d, 2 H, H2'6' phenyl) J 9 Hz, 7.69 (d, $1 \mathrm{H}$, H8 benzothiazine) $J 8.1 \mathrm{~Hz}, 7.84$ (s, $1 \mathrm{H}, \mathrm{CH}$ ethylene), 7.94 (dd, $1 \mathrm{H}, \mathrm{H} 7$ benzothiazine) $J 10.5$ and $2.1 \mathrm{~Hz}, 7.96(\mathrm{~d}, 1 \mathrm{H}, \mathrm{H} 5$ benzothiazine) $J 1.8 \mathrm{~Hz}$; MS, $m / z(\%)$ : 342(M+24,85), 314(5.75), 310(58.9), 308(100), 306(30.7), 296(9.5), 292(14.6).

(2Z)-6-Amino-2-(4-methoxybenzylidene)-4H-benzo[1,4]thiazin3-one (9)

Over a period of $15 \mathrm{~min}, 100 \mathrm{mg}(0.3 \mathrm{mmol})$ of compound (7) was added stepwise to a cold, stirring solution of $324 \mathrm{mg}(0.3 \mathrm{mmol})$ of stannous dihydrate in $35 \mu \mathrm{L}$ of concentrated $\mathrm{HCl}$. The mixture was then left for $15 \mathrm{~min}$ at room temperature and refluxed for $2 \mathrm{~h}$. After cooling, the precipitate was suspended in water and a $20 \%$ sodium hydroxide solution was added ( $\mathrm{pH} 10)$ to produce the corresponding amine, which was collected and washed with $10 \%$ sodium hydroxide and water. $\mathrm{C}_{16} \mathrm{H}_{14} \mathrm{~N}_{2} \mathrm{O}_{2} \mathrm{~S}, 0.061 \mathrm{~g}(68 \%), \mathrm{mp} 210-213{ }^{\circ} \mathrm{C}$; TLC silica gel plates type $60 \mathrm{~F}_{254}, \mathrm{R}_{\mathrm{f}}\left(\mathrm{CHCl}_{3}: \mathrm{EtOH} 9: 1\right)$ 0.58; IR (potassium bromide) $v_{\max } \mathrm{cm}^{-1}: 3387,3302,3017,2958,2835,1659,1604,1509$, 1253, 1173, 801. ${ }^{1} \mathrm{H}$ NMR (300 MHz, DMSO-d $)$ ): $\delta 3.81(\mathrm{~s}, 3 \mathrm{H}$, $\left.\mathrm{OCH}_{3}\right), 5.24$ (s, $\left.2 \mathrm{H}, \mathrm{NH}_{2}\right), 6.26$ (d, $1 \mathrm{H}, \mathrm{H} 5$ benzothiazine) $\mathrm{J} 2.1 \mathrm{~Hz}$, 6.28 (d, $1 \mathrm{H}, \mathrm{H} 7$ benzothiazine) $J 8.4 \mathrm{~Hz}, 6.90$ (d, $1 \mathrm{H}, \mathrm{H} 8$ benzothiazine) J 8.1 Hz, 7.05 (d, 2 H, H3' 5' phenyl) J 9 Hz, 7.63 (d, 2 H, H2' 6' phenyl) $J 9 \mathrm{~Hz}, 7.67$ (s, $1 \mathrm{H}, \mathrm{CH}$ ethylene); MS, $m / z(\%): 299\left(\mathrm{MH}^{+}\right.$ 14.34), 297(84.7), 265(100), 250(22.9), 237(13.1), 222(17.3).

\section{(2Z)-6-Butylamino-2-(4-methoxybenzylidene)-4-methyl-4H-} benzo[1,4] thiazin-3-one (12)

We successfully used the method of condensation described for (7). $\mathrm{C}_{21} \mathrm{H}_{24} \mathrm{~N}_{2} \mathrm{O}_{2} \mathrm{~S}, 0.055 \mathrm{~g}(50 \%), \mathrm{mp} 102-104^{\circ} \mathrm{C}$; TLC silica gel plates type $60 \mathrm{~F}_{254}, \mathrm{R}_{\mathrm{f}}\left(\mathrm{CHCl}_{3}: \mathrm{EtOH} 9: 1\right) 0.47$; IR (KBr) $v_{\max } \mathrm{cm}^{-1}: 3371$, 2958, 2926, 1635, 1601, 1507, 1257, 1143, 827. ${ }^{1} \mathrm{H}$ NMR (300 MHz, DMSO-d $)_{6}: \delta 0.91$ (t, $\left.3 \mathrm{H}, \mathrm{CH}_{3} \delta\right) J 7.2 \mathrm{~Hz}, 1.31-1.44\left(\mathrm{~m}, 2 \mathrm{H}, \mathrm{CH}_{2}\right.$ ß), 1.48-1.57 (m, $\left.2 \mathrm{H}, \mathrm{CH}_{2} \gamma\right), 2.98-3.04\left(\mathrm{~m}, 2 \mathrm{H}, \mathrm{CH}_{2} \alpha\right), 3.41$ (s, 3 $\left.\mathrm{H}, \mathrm{NCH}_{3}\right), 3.81\left(\mathrm{~s}, 3 \mathrm{H}, \mathrm{OCH}_{3}\right), 5.79(\mathrm{t}, 1 \mathrm{H}, \mathrm{NH}) J 5.6 \mathrm{~Hz}, 6.33$ (dd, 
$1 \mathrm{H}, \mathrm{H} 7$ benzothiazine) $J 8.4$ and $2.1 \mathrm{~Hz}, 6.43$ (d, $1 \mathrm{H}, \mathrm{H} 5$ benzothiazine) $J 2.1 \mathrm{~Hz}, 7.02$ (d, $1 \mathrm{H}, \mathrm{H} 8$ benzothiazine) $J 8.7 \mathrm{~Hz}, 7.05$ (d, 2 H, H3'5' phenyl) J 9 Hz, 7.63 (d, 2 H, H2' 6' phenyl) J 9 Hz, 7.69 (s, $1 \mathrm{H}, \mathrm{CH}$ ethylene); $\mathrm{MS}, \mathrm{m} / z(\%): 368\left(\mathrm{M}^{+} 43.89\right), 367(100), 335(28)$, 334(20.1), 326(13.3), 312(10.2), 309(11.6), 256(8.5).

\section{SUPPLEMENTARY INFORMATION}

Supplementary crystallographic data have been deposited in the Cambridge Crystallographic Data Center as supplementary publication No. CCDC 242133. Copies of the available material can be obtained, free of charge, by contacting the Director at CCDC, 12 Union Road, Cambridge CH21EZ, UK (fax: +44-1223-336-033 or by e-mail: deposit@ccdc.cam.ac.uk or http://www.ccdc.cam.ac.uk.

\section{ACKNOWLEDGEMENTS}

To Prof. J-J. Bourguignon of Strasbourg University for his contribution to the development of this work. This work was supported by the CNPq and CAPES Brazilian national agencies.

\section{REFERENCES}

1. Fringuelli, R.; Milanese, L.; Schiaffella, F.; Rev. Med. Chem. 2005, 5, 1061.

2. Gupta, R. R.; Ojha, K .G. In Phenothiazines and 1,4-Benzothiazines: Chemical and Biochemical Aspects; Gupta, R. R., ed.; Elsevier: Amsterdam, 1988, p. 163-269.

3. Grandolini, G.; Ambrogi, V.; Rossi, C.; Tiralti, M.C.; Tuttobello, L.; Eur. J. Med. Chem. 1986, 21, 455.

4. Gupta, R. R.; Dev, P. K.; Sharma, M. L.; Rajoria, C. M.; Gupta, A.; Nyati, M.; Anti-cancer Drugs 1993, 4, 589.
5. Guarda, V. L. M.; Perrissin, M.; Thomasson, F.; Ximenes, E. A.; Galdino, S. L.; Pitta, I. R.; Luu-Duc, C.; Heterocycl. Commun. 2000, 6, 49.

6. Guarda, V. L. M.; Pereira, M. A.; De Simone, C. A.; Albuquerque, J. F. C.; Galdino, S. L.; Chantegrel, J.; Perrissin, M.; Beney, C.; Thomasson, F.; Pitta, I. R.; Luu-Duc, C.; Sulfur Lett. 2003, 26, 17.

7. Silva, T. G.; Barbosa, F. S. V.; Brandão, S. S. F.; Lima, M. C. A.; Galdino, S. L.; Pitta, I. R.; Barbe, J.; Heterocycl. Commun. 2001, 7, 523.

8. Souza, A. M. A.; Guarda, V. L. M.; Leite, L. F. C.; Barbosa Filho, J. M.; Lima, M. C. A.; Galdino, S. L.; Pitta, I. R.; Quim. Nova 2006, 29, 1106.

9. Ngadi, L.; Galy, A. M.; Galy, J. P.; Barbe, J.; Crémieux, A.; Chevalier, J.; Eur. J. Med. Chem. 1990, 25, 67.

10. Cecchetti, V.; Dominici, S.; Fravolini, A.; Schiaffella, F.; Eur. J. Med. Chem. 1984, 19, 29.

11. Krapcho, J.; Turk, C. F.; J. Med. Chem. 1973, 16, 776.

12. Brandão, S. S. F.; Guarda, V. L. M.; Chantegrel, J.; Perrissin, M.; Galdino, S. L.; Thomasson, F.; Souza, V. M.; Lima, M. C. A.; Leite, L. F. C. C.; Pitta, I. R.; Luu-Duc, C.; Boll. Chim. Farm. 2000, 139, 54.

13. Enraf-Nonius; Difractometer Kappa - CCD, Nonius BV; Delft ; The Netherlands, 1999.

14. Otwinowski, Z.; Minor, W.; Denzo, H. K. In Methods in Enzymology, 276; Carter, Jr., C. W.; Sweet, R. M., eds.; Academic Press: New York, 1997 , p. 307-326.

15. Enraf-Nonius; COLLECT; Nonius BV, Delft: The Netherlands, 19972000.

16. Sheldrick, G. M.; SHELXS-97. Program for Crystal Structure Resolution, Univ. of Göttingen: Göttingen, Germany, 1997a.

17. Sheldrick, G. M.; SHELXL-97. Program for Crystal Structures Analysis, Univ. of Göttingen: Göttingen, Germany, 1997b.

18. Farrugia, L. J.; J. Appl. Cryst. 1997, 30, 565.

19. Farrugia, L. J.; J. Appl. Cryst. 1999, 32, 837. 